\title{
Gastrectomy with or without neoadjuvant S-1 plus cisplatin for type 4 or large type 3 gastric cancer (JCOG0501): an open-label, phase 3, randomized controlled trial
}

\author{
Yoshiaki Iwasaki ${ }^{1} \cdot$ Masanori Terashima $^{2}$ - Junki Mizusawa ${ }^{3} \cdot$ Hiroshi Katayama $^{3} \cdot K^{2}$ enichi Nakamura ${ }^{3}$. \\ Hitoshi Katai ${ }^{4}$. Takaki Yoshikawa ${ }^{5}$ - Seiji Ito ${ }^{6}$. Masahide Kaji ${ }^{7}$ Yutaka Kimura ${ }^{8} \cdot$ Motohiro Hirao $^{9} \cdot$ Makoto Yamada $^{10}$. \\ Akira Kurita $^{11}$ - Masakazu Takagi ${ }^{12}$. Sang-Woong Lee ${ }^{13} \cdot$ Akinori Takagane $^{14} \cdot$ Hiroshi Yabusaki $^{15}$. Jun Hihara ${ }^{16}$. \\ Narikazu Boku ${ }^{17} \cdot$ Takeshi Sano $^{18} \cdot$ Mitsuru Sasako $^{19}$
}

Received: 3 September 2020 / Accepted: 19 October 2020 / Published online: 16 November 2020

(c) The International Gastric Cancer Association and The Japanese Gastric Cancer Association 2020

\begin{abstract}
Background Specific treatment strategies are sorely needed for scirrhous-type gastric cancer still, which has poor prognosis. Based on the promising results of our previous phase II study (JCOG0210), we initiated a phase III study to confirm the efficacy of neoadjuvant chemotherapy (NAC) in type 4 or large type 3 gastric cancer.

Methods Patients aged 20-75 years without a macroscopic unresectable factor as confirmed via staging laparoscopy were randomly assigned to surgery followed by adjuvant chemotherapy with S-1 (Arm A) or NAC (S-1plus cisplatin) followed by D2 gastrectomy plus adjuvant chemotherapy with S-1 (Arm B). The primary endpoint was overall survival (OS).

Results Between October 2005 and July 2013, 316 patients were enrolled, allocating 158 patients to each arm. In Arm B, in which NAC was completed in $88 \%$ of patients. Significant downstaging based on tumor depth, lymph node metastasis, and peritoneal cytology was observed using NAC. Excluding the initial 16 patients randomized before the first revision of the protocol, 149 and 151 patients in arms A and B, respectively, were included in the primary analysis. The 3-year OS rates were 62.4\% [95\% confidence interval (CI) 54.1-69.6] in Arm A and 60.9\% (95\% CI 52.7-68.2) in Arm B. The hazard ratio of Arm B against Arm A was 0.916 (95\% CI 0.679-1.236).

Conclusions For type 4 or large type 3 gastric cancer, NAC with S-1 plus cisplatin failed to demonstrate a survival benefit. D2 surgery followed by adjuvant chemotherapy remains the standard treatment.
\end{abstract}

Keywords Type $4 \cdot$ Linitis plastica $\cdot$ Large type $3 \cdot$ Neoadjuvant chemotherapy

\section{Introduction}

Gastric cancer (GC) is the third leading cause of cancerrelated death [1]. Although the incidence of GC in Japan has decreased in recent years, similar to that observed in Western countries, it remains the second most common cancer in Japan [2]. Among GCs, Borrmann type 4 GC, including linitis plastica and scirrhous-type cancer, has the

Electronic supplementary material The online version of this article (https://doi.org/10.1007/s10120-020-01136-7) contains supplementary material, which is available to authorized users.

Masanori Terashima

m.terashima@scchr.jp

Extended author information available on the last page of the article unique characteristics of diffuse invasion inside the gastric wall, easy spread to the peritoneum, and extremely poor prognosis even after curative D2 gastrectomy. Large ulceroinvasive type (type 3) GC that measures more than $8 \mathrm{~cm}$ in diameter has similar biological characteristics as type $4 \mathrm{GC}$ [3]. Pedrazzani et al. reported the limited role of surgery in these types of cancer and recommended the development of a multimodal treatment strategy [4].

To improve the prognosis of these aggressive types of GC, neoadjuvant chemotherapy may be a preferable approach in terms of the eradication of micrometastasis in addition to local control, higher compliance with intensive chemotherapy, and avoidance of futile surgery by detection of initially invisible distant metastasis after rapid disease progression during neoadjuvant chemotherapy. As for the NAC regimen, S-1 plus cisplatin (SP), which is a standard 
first-line chemotherapy for metastatic disease associated with high response rates and acceptable toxicities [5], was considered the most promising candidate. Therefore, the Stomach Cancer Study Group of the Japan Clinical Oncology Group (SCSG/JCOG) initiated a multi-institutional phase II study named JCOG0210 to evaluate the efficacy and safety of NAC with SP followed by D2 gastrectomy for these special GC types in 2003. The completion proportion of the protocol treatment, which was the primary endpoint, was $73.5 \%$ [80\% confidence interval (CI) 63.7-81.7), exceeding the prespecified threshold of $45 \%(P<0.0001)$. Additionally, the rate of treatment-related deaths was $2.0 \%$ (1/49), which was lower than the prespecified threshold $(<5 \%)$ [6].

Based on these data, we conducted a phase III study, JCOG0501, to confirm the superiority of neoadjuvant SP followed by D2 gastrectomy over upfront surgery. The shortterm safety and surgical results of this phase III trial have been reported previously [7]. Here, we report the results of the primary analysis of JCOG0501, focusing on overall survival.

\section{Methods}

\section{Patients}

The eligibility criteria for this study were as follows: (1) histologically proven adenocarcinoma of the stomach; (2) Borrmann type 4 or large ( $\geq 8 \mathrm{~cm}$ ) type 3 ; (3) no evidence of distant metastasis; (4) no involvement of the esophagus $>3 \mathrm{~cm}$; (5) age of 20-75 years; (6) an Eastern Cooperative Oncology Group performance status of 0 or 1 ; (7) no previous chemotherapy or radiotherapy for any malignancy; (8) no previous surgery for $\mathrm{GC}$ excluding endoscopic mucosal resection or endoscopic submucosal dissection; (9) no prominent bleeding from the primary tumor or gastrointestinal stenosis; (10) sufficient oral intake; and (11) adequate organ function (white blood cell count, $>3000$ and $<12000 / \mathrm{mm}^{3}$; hemoglobin, $>9.0 \mathrm{~g} / \mathrm{dl}$; platelet count, $>100000 / \mathrm{mm}^{3}$; aspartate aminotransferase and alanine aminotransferase, $<100 \mathrm{IU}$; total bilirubin, $<2.0 \mathrm{mg} / \mathrm{dl}$; creatinine, $<1.5 \mathrm{mg} / \mathrm{dl}$; and creatinine clearance, $>60 \mathrm{ml} / \mathrm{min})$. Written informed consent was obtained from all patients prior to their participation in the study.

Tumors were staged in accordance with the 13th Japanese Classification of Gastric Carcinoma (corresponding to the 2nd English edition) [8]. Initially, in this study, exploratory laparoscopy and peritoneal lavage cytology were mandatory to exclude patients with positive peritoneal cytology for carcinoma cells (CY1) and those with peritoneal metastases. However, after the second protocol revision due to the poor accrual reflecting the rarity of $\mathrm{p} 0 / \mathrm{CY} 0$ disease within type 4 and large type 3 GCs, patients with CY 1 and those with dissemination only to the peritoneum adjacent to the stomach (classified as P1 in the $1^{\text {st }}$ English Edition of the Japanese Classification of Gastric Carcinoma [9]) became eligible (September, 2009).

The exclusion criteria were as follows: (1) synchronous or metachronous (within 5 years) malignancy other than carcinoma in situ; (2) pregnant or lactating women; (3) treatment with a major tranquilizer, steroids, flucytosine, phenytoin, or warfarin; and (4) lung fibrosis, interstitial pneumonitis, bowel obstruction, or ischemic heart disease.

\section{Study design}

This was an open-label, randomized phase III trial (JCOG0501) conducted by the SCSG in JCOG. Eligible patients were registered by telephone or fax to the JCOG Data Center. Patients were randomly assigned (1:1) to undergo upfront surgery (Arm A) or NAC followed by surgery (Arm B). A minimization method with a random component was used to balance the arms based on institution, macroscopic type (large type 3 vs. type 4), clinical depth of invasion (T2/3 vs. T4), and clinical nodal status (N0 vs. $\mathrm{N} 1$ vs. N2). All patients and investigators were unmasked to treatment assignment. The JCOG Data Center performed central monitoring to ensure data submission, patient eligibility, protocol compliance, safety, and on-schedule study progress and issued monitoring reports every six months.

The primary endpoint was overall survival (OS), defined as the time from randomization to death from any cause or the last date of contact for surviving patients. Concerning the secondary endpoints, progression-free survival (PFS) was defined as the time from randomization to the first occurrence of disease progression confirmed by clinical or image diagnosis, such as progression before surgery, diagnosis of being unable to undergo $\mathrm{R} 0$ or $\mathrm{R} 1$ resection even when a progression-free status was verified, or death from any cause. Thus, positive peritoneal cytology or peritoneal metastasis incidentally found during surgery was not regarded as an event if $\mathrm{R} 0$ or $\mathrm{R} 1$ resection could be performed. Protocol completion in Arm B was defined the completion of NAC and achievement of $\mathrm{R} 0$ resection with D2 or D3 (dissection of No.13 and 14v in addition to the D2 dissection for distal cancer) or greater lymph node dissection. The proportion of $\mathrm{R} 0$ resection was defined as the proportion of patients achieving $\mathrm{R} 0$ resection with $\mathrm{D} 2 / 3$ lymph node dissection (by the Japanese Classification of Gastric Carcinoma, 2nd English Edition). Response to NAC in the primary lesion was evaluated according to the clinical criteria for the response assessment of chemotherapy and radiotherapy for gastric carcinoma: clinical criteria [11]. Adverse events (AEs) associated with either gastrectomy or 
chemotherapy were separately evaluated using the Common Terminology Criteria for Adverse Events (version 3.0).

The study protocol was approved by the JCOG Protocol Review Committee and the institutional review board of each participating hospital before initiation of the study. This study was performed in accordance with the international ethical recommendations stated in the Declaration of Helsinki and the Japanese Ethical Guidelines for Clinical Research.

\section{Treatment}

Patients assigned to Arm A underwent total or distal gastrectomy with D2/3 lymph node dissection depending on the tumor location. Patients assigned to Arm B received NAC with SP before surgery. S-1 was administered orally twice daily for the first 3 weeks of a 4 -week course. The actual dose of $\mathrm{S}-1$ ( $80 \mathrm{mg} / \mathrm{m}^{2}$ per day) was adjusted by body surface area: $<1.25 \mathrm{~m}^{2}, 80 \mathrm{mg} ; 1.25-1.5 \mathrm{~m}^{2}, 100 \mathrm{mg}$; and $>1.5 \mathrm{~m}^{2}$, $120 \mathrm{mg}$. Cisplatin $\left(60 \mathrm{mg} / \mathrm{m}^{2}\right)$ was administered via intravenous infusion on day 8 of each course, as described previously [6]. Tumor resectability was assessed using computed tomography, upper gastroenterological endoscopy, and barium meal study 14-20 days after the termination of the second course of chemotherapy. The resection criteria were as follows: (i) R0 resection was deemed possible by gastrectomy with D2 or D3 according to the Japanese Classification of Gastric Carcinoma, 2nd English Edition) and (ii) sufficient organ function (white blood cell count $>3000 /$ $\mathrm{mm}^{3}$, platelet count $>100000 / \mathrm{mm}^{3}$ ). Patients who fulfilled these criteria underwent surgery within 42 days after the last chemotherapy dose. Surgery in group B was performed as described for Arm A.

After the first protocol revision, adjuvant chemotherapy, comprising eight courses of S-1, was started within 6 weeks after surgery for both arms. All patients who underwent curative resection received S-1 orally on days 1-28 of each 6-week cycle. Treatment was discontinued in patients with disease progression based on diagnosis by clinical assessment or imaging studies, in patients with AEs (serious AEs, those causing treatment cycle delays for longer than 4 weeks, or those requiring subsequent dose reduction after the second dose reduction), patient refusal, or judgment by the attending physician for other reasons.

\section{Statistical analysis}

This study was designed to confirm the superiority of Arm B compared with Arm A in terms of OS. The planned sample size was 300 (150 per arm), expecting a total of 276 deaths, to detect a 3-year survival difference of $10 \%$ [17.5\% in Arm
A vs. $27.5 \%$ in Arm B, corresponding to a hazard ratio (HR) of 0.74 , with a one-sided $\alpha$ of $5 \%$ and $80 \%$ statistical power. Follow-up for 3 years was planned after 5 years of patient accrual.

The protocol was revised to add adjuvant chemotherapy with S-1 for 1 year in both arms on February 13, 2007 based on the results of the ACTS-GC trial [3]. The full analysis set for efficacy and safety was defined as the total patients enrolled after this protocol revision. At this time, 16 patients had already been enrolled. Taking the survival benefit by adjuvant chemotherapy into consideration, the sample size of the full analysis set was recalculated as 300, after excluding the 16 already enrolled patients, based on a 3-year survival difference of $10.8 \%$ (25\% in Arm A vs. 35.8\% in Arm $\mathrm{B}$, corresponding to $\mathrm{HR}=0.74$ ) with a one-sided $\alpha$ of 5 and $80 \%$ statistical power.

Because of slow accrual, the protocol was revised again to include P0CY1, P1CY0, and P1CY1 diagnosed at staging laparoscopy on July 6, 2009. The sample size was unchanged, because the estimated prognosis of these patients was considered identical to that of the originally eligible patients with P0CY0 according to staging laparoscopy.

Two interim analyses were planned with adjustments for repeated comparisons using the Lan and DeMets method and the O'Brien-Fleming type $\alpha$ spending function [10]. The first and second interim analyses were planned when half and all of the planned accrual was completed, respectively.

OS and PFS were estimated using the Kaplan-Meier method. The primary analysis of OS was performed using the stratified log-rank test with stratification based on the macroscopic type and clinical nodal status. HRs and CIs for OS were estimated using the stratified Cox proportional hazards model analysis with macroscopic type and clinical nodal status as the strata. The unstratified log-rank test and unstratified Cox proportional hazards model were used for PFS. Prespecified subgroup analyses were conducted using the following variables for OS: age, sex, macroscopic type, histology, clinical depth of invasion, clinical nodal status, and cytology. Although the sample size was estimated based on a one-sided alpha of 5\%, the two-sided $95 \%$ CI was provided. The $P$ value for OS was one-sided. Other $P$ values were two-sided. Statistical analyses were performed by the JCOG Data Center using the SAS software program version 9.4 (Cary, NC, USA). The study was registered with UMINCTR (No. C000000279).

\section{Results}

Between October 17, 2005 and July 19, 2013, 316 patients were randomly assigned to Arm A (158 patients) or Arm B (158 patients) at 44 hospitals in Japan (Fig. 1). Two patients in Arm B were deemed ineligible. The final 


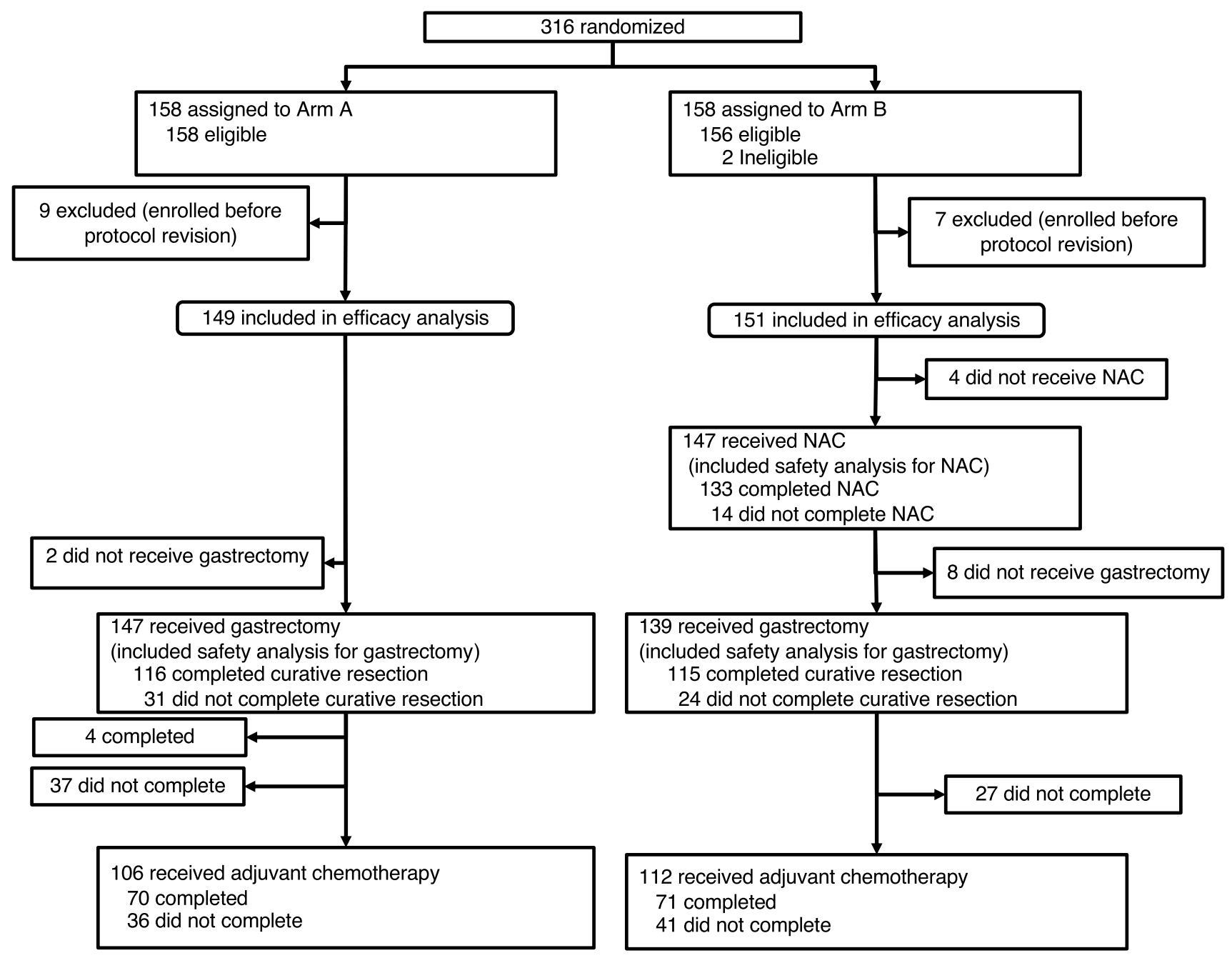

Fig. 1 Flow diagram of patient enrollment and randomization. Cx, chemotherapy

pathological diagnosis of the resected specimen was malignant lymphoma in one patient, and another patient had peritoneal metastasis diagnosed as P2 at the time of staging laparoscopy. These two patients were included in the efficacy analysis.

Nine patients in Arm A and seven patients in Arm B were enrolled before the first protocol revision, and thus, they were excluded from the efficacy and safety analyses. The patient characteristics of the full analysis set were balanced between the arms (Table 1). Type 3 accounted for one-third of all malignancies in both arms, and the remaining twothirds of tumors were type 4 . The cT3 (SE) and undifferentiated tumor types were predominant in both arms. Approximately $60 \%$ of the patients had signet-ring cell component in both arms. Limited peritoneal metastasis (P1), which was included in this study after the second revision, was found in one and four patients in arms A and B, respectively, at the time of staging laparoscopy. Peritoneal cytology was positive in 28 patients in Arm A and in 32 patients in Arm B.
The first and second interim analyses were performed on September 17 and March 15, 2014, respectively. In both interim analyses, the Data and Safety Monitoring Committee recommended the continuation of the study.

\section{Neoadjuvant chemotherapy}

Of 151 patients eligible for efficacy analysis in Arm B, four patients did not receive NAC because of patient refusal, acute cholecystitis, liver dysfunction, and miscellaneous reasons in one patient each. As a result, 147 patients in Arm $B$ who received NAC were included for this safety analysis. Among these 147 patients, NAC was terminated during the first course in 12 patients and during the second course in two patients, and the remaining 133 patients completed two courses of NAC (88\%). The reasons for termination of NAC were AEs, patient refusal, and disease progression in eight, four, and two patients, respectively. The relative dose 
intensities of cisplatin and S-1 during NAC [median (quartile)] were $96.4 \%(80.3 \%-100 \%)$ and $90.0 \%(81.5 \%-100 \%)$, respectively.

Details of AEs have been published previously [7]. The major grade 3 or grade 4 toxicities during NAC in the 147 patients included neutropenia (29.3\%), leukopenia $(7.5 \%)$, anemia $(4.1 \%)$, anorexia $(11.6 \%)$, hyponatremia $(6.8 \%)$, nausea $(5.4 \%)$, diarrhea (4.8\%), and vomiting (2.7\%). Clinical response of the primary tumor was assessed in all 151 patients of the Arm B. Based on the criteria, partial response, stable disease, and progressive disease were noted in 50, 60, and 4 patients, respectively, whereas the results were not evaluable and missing for 18 and 19 patients, respectively. Thus, the macroscopic response rate was $33.1 \%$.

\section{Surgical resection}

In Arm A, one patient did not undergo gastrectomy because of disease progression, and one patient received NAC after refusing upfront surgery (Fig. 1). In Arm B, eight patients did not undergo gastrectomy as follows: because of disease progression in five patients (two after NAC and three during NAC), refusal in one patient, AEs caused by NAC in one patient, and death caused by another disease in one patient. The type of surgery and the presence or absence of combined resection were not markedly different between the arms, as described in detail in the previous publication [7]. The protocol completion rate defined in the protocol in Arm B was $68.2 \%$ (95\% CI 60.2-75.5).

\section{Pathological findings}

The pathological findings were available in 147 and 139 patients in arms A and B, respectively, as shown in Table 2. There were significant differences in tumor depth (pT), lymph node metastasis $(\mathrm{pN})$, peritoneal cytology (CY), and pathological stage between the arms. This may indicate that neoadjuvant chemotherapy with SP downstages resectable type 4 or large type $3 \mathrm{GC}$ in terms of T, N status and CY. The pathological response rate in 151 patients, defined by degeneration/necrosis in more than one-third of the tumor (Grades 1b, 2, and 3), was 51\% (77/151). Pathological complete response (pCR) was observed in only three patients $(2.0 \%$, Table 3$)$.

\section{Adjuvant chemotherapy}

Adjuvant chemotherapy was initiated in 106 and 112 patients in arms A and B, respectively. The adjuvant chemotherapy completion rate was 70/106 (66.0\%) in Arm A and $71 / 112(63.4 \%)$ in Arm B. The completion rates of planned treatment were 74/149 (49.7\%) and 71/151 (47.0\%) in Arms $\mathrm{A}$ and $\mathrm{B}$, respectively.

\section{OS and PFS}

During a median follow-up period of 4.5 years including 300 patients, 91 and 88 patients died and 89 and 91 patients experienced disease progression in Arms A and B, respectively. Figure $2 \mathrm{a}$, b presents the OS and PFS of 300 patients. The OS and PFS of both arms were considerably better than those reported previously, and the survival curves were almost similar between the arms. The 3-year OS rate was 62.4\% (95\% CI 54.1-69.6) in Arm A, vs. 60.9\% (52.7-68.2) in Arm B. The HR for Arm B against Arm A was 0.916 (95\% CI 0.679-1.236, one-sided $P=0.28$ ). The 3 -year PFS rate was $47.7 \%$ in both arms, with a $95 \%$ CI of 39.4-55.4 in Arm A and 39.5-55.4 in Arm B. The HR was 0.976 (95\% CI $0.738-1.292, P=0.87)$. The OS was not different when 16 patients who were excluded at the time of the first protocol amendment were added to the analysis (Supplemental Fig. 1).

We also conducted subgroup analyses (Fig. 3). HR was almost similar in all subcategories (0.83-0.96) excluding histological type, clinical nodal status, and peritoneal cytology in staging laparoscopy. An interaction was noted for histological type. HRs classified by histology were $0.69(95 \%$ CI 0.41-1.14) for non-signet ring cell (SRC) type $(n=123)$ and 1.16 (95\% CI $0.81-1.67)$ for SRC type $(n=176)$ ( $P=0.098$ for interaction).

Table 3 lists the sites of progression in all patients included in the primary analysis. The most frequent site was the peritoneum $(77.3 \%)$ in both arms, and the pattern of progression did not differ according to the receipt of NAC.

\section{Discussion}

This phase III trial (JCOG0501) examined whether the addition of NAC with SP could improve the survival of resectable type 4 or large type $3 \mathrm{GC}$ compared with upfront surgery. However, the results did not confirm the effectiveness of NAC with SP. Compared to the results (3-year OS rate $=24.5 \%$ ) of the previous phase II study (JCOG0210) [6], when there was no established adjuvant chemotherapy, the current trial demonstrated excellent survival in both arms. Actually, the 3-year OS was more than doubled during these two decades. This high OS rate could be explained by the efficacy of adjuvant S-1 chemotherapy besides improvement in diagnosis and perioperative treatment for these types of tumors. It has been demonstrated that S-1 was effective in preventing peritoneal recurrence, which is the most frequently observed type of recurrence in type 4 and large type 


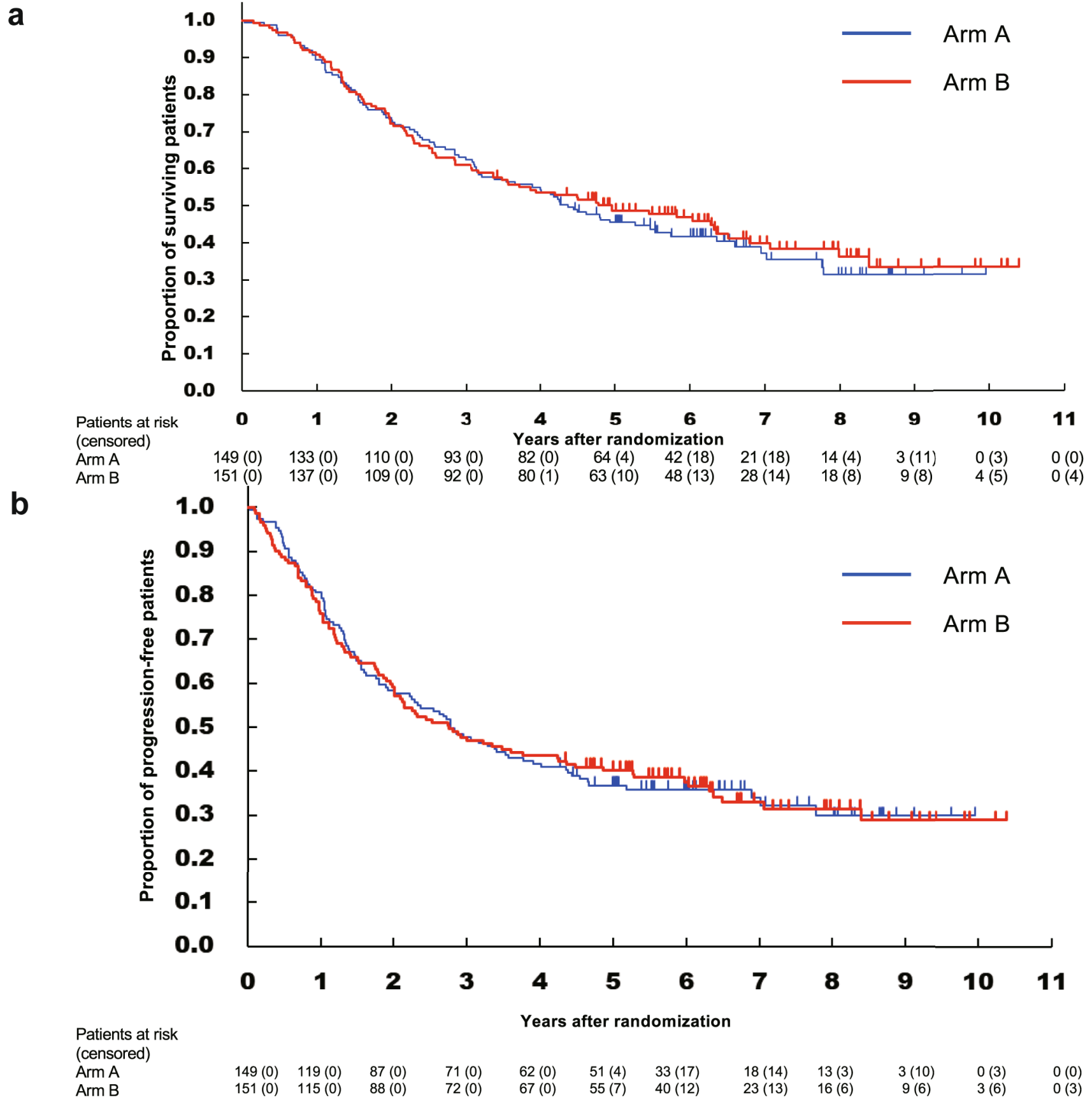

Fig. 2 Kaplan-Meier estimates of overall survival (a) and progression-free survival (b). a Overall survival in the surgery + adjuvant S-1 group (Arm A) vs. the neoadjuvant $\mathrm{SP}+$ surgery + adjuvant $\mathrm{S}-1$ group (Arm B). The hazard ratio for Arm B against Arm A was 0.916 [95\%

3 GCs [12]. This is a good example for the necessity of randomized controlled trials instead of single-arm trials with historical controls for the development of novel modality treatment.

In the present study, histological response was observed in approximately half of the patients who received NAC, confidence interval (CI) 0.679-1.236; one-sided $P=0.28$ ]. b Progression-free survival in the Arm A vs. Arm B. The hazard ratio for Arm B against Arm A was 0.976 (95\% CI 0.738-1.292; $P=0.87$ ).

and approximately one-third of the patients exhibited grade 2 (more than two-thirds) tumor shrinkage. In fact, obvious tumor downstaging was observed with NAC compared with upfront surgery in terms of T, N status, and CY. Accordingly, the proportion of $\mathrm{R} 0$ resection was significantly higher in patients treated with NAC than in those who did not 


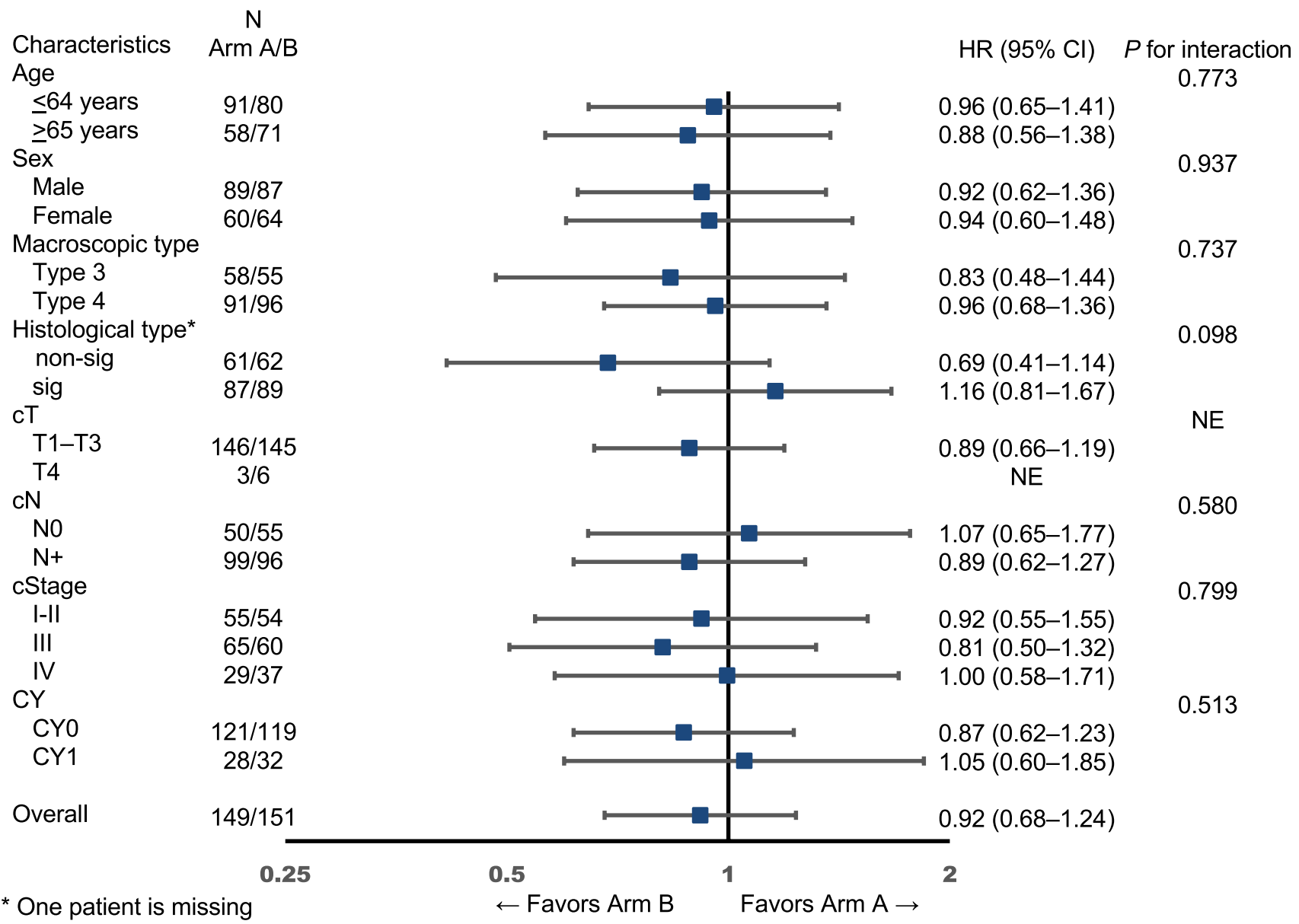

Fig. 3 Forest plots and the interpretation of subgroups for overall survival $(n=300)$. The forest plot presents hazard ratios (HRs) for death (oblongs) and 95\% confidence intervals (CIs, I bars). $P$ values indicate interactions between treatment and subgroup variables. *Signet-

receive NAC. However, the high tumor response rate did not translate to a survival benefit. Histological response has been reported to be associated with the improvement in survival in patients who receive NAC for GC [13]. However, this may not be true in patients with type 4 or large type 3 GC. Furthermore, it has been reported that conversion to negative peritoneal cytology did not result in improved survival in GC [14]. This result also supports the negative association between histological response and survival in the present study. The precise analysis of a potential association between histological response and survival should be conducted.

We evaluated primary tumor response using upper gastrointestinal X-ray according to the study protocol. Partial response was observed in 50 patients, resulting in the response rate of $33.3 \%$. Although none of the patients achieved complete response, three patients exhibited pathological response, which was consistent with previously published studies [6, 15]. Evaluation of macroscopic tumor response is difficult, especially in patients with type 4 GC. ring cell histology was diagnosed using pretreatment biopsy specimens. $c T$ clinical tumor stage, $c N$ clinical node stage, $C Y$ peritoneal cytology in staging laparoscopy, $N E$ not evaluable

In Western countries, perioperative epirubicin, cisplatin, and 5-fluorouracil (ECF) chemotherapy, a standard perioperative chemotherapy regimen, was widely accepted after positive results were obtained in the MAGIC trial [16]. Recently, the results of the FLOT4 trial revealed that the fluorouracil, leucovorin, oxaliplatin, and docetaxel (FLOT) regimen improved the outcome of resectable gastric and gastroesophageal junction (GEJ) cancer compared with perioperative ECF/epirubicin, cisplatin and capecitabine [17, 18]. Therefore, based on these results, NAC with FLOT4 regimens is regarded as a standard treatment for advanced gastric or GEJ cancer in Western countries as far as patient can tolerate the treatment. However, we failed to demonstrate the efficacy of NAC in type 4 and large type 3 GC. This is the first phase III trial assessing the efficacy of NAC for $\mathrm{GC}$ in East Asian countries. There are several reasons for the negative results of this study compared with the FLOT4 trial. The major possible reason is the difference in the proportion of $\mathrm{pCR}$ between the two trials. The reported $\mathrm{pCR}$ in 
Table 1 Patient characteristics $(n=300)$

\begin{tabular}{|c|c|c|c|c|}
\hline & \multicolumn{2}{|c|}{$\operatorname{Arm~A~}(n=149)$} & \multicolumn{2}{|c|}{$\begin{array}{l}\text { Arm B } \\
(n=151)\end{array}$} \\
\hline & $n$ & $(\%)$ & $n$ & $(\%)$ \\
\hline Age (years), median (range) & 62 & $(28-75)$ & 64 & $(30-75)$ \\
\hline \multicolumn{5}{|l|}{ Sex } \\
\hline Male & 89 & $(59.7)$ & 87 & $(57.6)$ \\
\hline Female & 60 & $(40.3)$ & 64 & $(42.4)$ \\
\hline \multicolumn{5}{|l|}{ ECOG PS } \\
\hline 0 & 141 & $(94.6)$ & 149 & $(98.7)$ \\
\hline 1 & 8 & $(5.4)$ & 2 & (1.3) \\
\hline \multicolumn{5}{|l|}{ Macroscopic type } \\
\hline Type 3 & 58 & $(38.9)$ & 55 & $(36.4)$ \\
\hline Type 4 & 91 & $(61.1)$ & 96 & $(63.6)$ \\
\hline \multicolumn{5}{|l|}{$\mathrm{cT}$} \\
\hline $\mathrm{cT} 2$ & 28 & $(18.8)$ & 17 & $(11.3)$ \\
\hline cT3 & 118 & $(79.2)$ & 128 & $(84.8)$ \\
\hline cT4 & 3 & $(2.0)$ & 6 & $(4.0)$ \\
\hline \multicolumn{5}{|l|}{$\mathrm{cN}$} \\
\hline $\mathrm{cNO}$ & 50 & $(33.6)$ & 55 & $(36.4)$ \\
\hline $\mathrm{cN} 1$ & 62 & $(41.6)$ & 67 & $(44.4)$ \\
\hline $\mathrm{cN} 2$ & 37 & $(24.8)$ & 29 & $(19.2)$ \\
\hline \multicolumn{5}{|l|}{ Histological type } \\
\hline Differentiated & 32 & $(21.5)$ & 39 & $(25.8)$ \\
\hline Undifferentiated & 117 & $(78.5)$ & 112 & $(74.2)$ \\
\hline \multicolumn{5}{|l|}{ Signet-ring cell component ${ }^{\mathrm{a}}$} \\
\hline Absent & 61 & $(40.9)$ & 62 & $(41.1)$ \\
\hline Present & 87 & $(58.4)$ & 89 & $(58.9)$ \\
\hline Unknown & 1 & $(0.7)$ & - & - \\
\hline \multicolumn{5}{|l|}{ Esophageal invasion } \\
\hline Absence & 135 & $(90.6)$ & 143 & $(94.7)$ \\
\hline Presence & 14 & $(9.4)$ & 8 & (5.3) \\
\hline \multicolumn{5}{|l|}{ Peritoneal metastasis } \\
\hline $\mathrm{P} 0$ & 148 & $(99.3)$ & 146 & $(96.7)$ \\
\hline $\mathrm{P} 1$ & 1 & $(0.7)$ & 5 & (3.3) \\
\hline \multicolumn{5}{|l|}{ Peritoneal cytology } \\
\hline CY0 & 121 & $(81.2)$ & 119 & $(78.8)$ \\
\hline CY1 & 28 & $(18.8)$ & 32 & $(21.2)$ \\
\hline \multicolumn{5}{|l|}{ cStage } \\
\hline IB & 12 & (8.0) & 6 & $(4.0)$ \\
\hline II & 43 & $(28.9)$ & 48 & $(31.8)$ \\
\hline IIIA & 41 & $(27.5)$ & 40 & $(26.5)$ \\
\hline IIIB & 24 & $(16.1)$ & 20 & $(13.2)$ \\
\hline IV & 29 & $(19.5)$ & 37 & $(24.5)$ \\
\hline
\end{tabular}

ECOG PS Eastern Cooperative Oncology Group performance status, $c T$ clinical tumor status, $c N$ clinical nodal status, $c$ Stage clinical stage ${ }^{\text {a }}$ Signet-ring cell component was identified using biopsy specimens

GC is less than $10 \%$ [19]. In fact, the proportion of pCR was only $2.0 \%$ in the present study. In contrast, the proportion of pCR in the FLOT4 trial was as high as $16 \%$ [17]. The high
Table 2 Pathological findings

\begin{tabular}{|c|c|c|c|c|c|}
\hline & \multicolumn{2}{|c|}{ Arm A $(N=147)$} & \multicolumn{2}{|c|}{ Arm B $(N=139)$} & \multirow[t]{2}{*}{$P$-value } \\
\hline & $n$ & $(\%)$ & $n$ & $(\%)$ & \\
\hline \multicolumn{6}{|l|}{ pT } \\
\hline$\leq \mathrm{T} 1$ & 2 & (1.4) & $9^{b}$ & $(6.5)$ & \multirow[t]{5}{*}{$<0.0001$} \\
\hline $\mathrm{T} 2$ & 29 & $(19.7)$ & 58 & $(41.7)$ & \\
\hline $\mathrm{T} 3$ & 99 & $(67.3)$ & 60 & $(43.2)$ & \\
\hline $\mathrm{T} 4$ & 14 & $(9.5)$ & 6 & $(4.3)$ & \\
\hline $\mathrm{Tx}$ & 3 & (3) & 6 & $(4.3)$ & \\
\hline \multicolumn{6}{|l|}{$\mathrm{pN}$} \\
\hline N0 & 19 & $(12.9)$ & 52 & $(37.4)$ & \multirow[t]{5}{*}{$<0.0001$} \\
\hline N1 & 40 & $(27.2)$ & 44 & $(31.7)$ & \\
\hline $\mathrm{N} 2$ & 70 & $(47.6)$ & 34 & $(24.5)$ & \\
\hline N3 & 15 & $(10.2)$ & 3 & $(2.2)$ & \\
\hline $\mathrm{Nx}$ & 3 & $(2.0)$ & 6 & (4.3) & \\
\hline \multicolumn{6}{|l|}{$\mathrm{pP}$} \\
\hline P0 & 122 & $(83.0)$ & 118 & (84.9) & \multirow[t]{2}{*}{0.6622} \\
\hline $\mathrm{P} 1$ & 25 & $(17.0)$ & 21 & $(15.1)$ & \\
\hline \multicolumn{6}{|l|}{ CY } \\
\hline CY0 & 107 & $(72.8)$ & 120 & $(86.3)$ & \multirow[t]{3}{*}{0.0148} \\
\hline CY1 & 39 & $(26.5)$ & 19 & $(13.7)$ & \\
\hline CYx & 1 & $(0.7)$ & 0 & & \\
\hline \multicolumn{6}{|l|}{ pStage } \\
\hline$\leq \mathrm{I}$ & 8 & (5.4) & $32^{\mathrm{b}}$ & $(23.0)$ & \multirow[t]{4}{*}{$<0.0001$} \\
\hline II & 18 & $(12.2)$ & 38 & $(27.3)$ & \\
\hline III & 61 & $(41.5)$ & 37 & $(26.6)$ & \\
\hline IV & 60 & $(40.8)$ & 32 & $(23.0)$ & \\
\hline \multicolumn{6}{|l|}{$\mathrm{R}$} \\
\hline R0 & 98 & $(66.6)$ & 112 & $(80.6)$ & \multirow[t]{3}{*}{0.0045} \\
\hline $\mathrm{R} 1$ & 35 & $(23.8)$ & 13 & & \\
\hline $\mathrm{R} 2$ & 14 & $(9.5)$ & 14 & & \\
\hline \multicolumn{6}{|c|}{ Pathological response $^{\mathrm{a}}$} \\
\hline Grade 0 & - & & 15 & $(10.8)$ & \multirow[t]{5}{*}{ N.A. } \\
\hline Grade 1a & - & & 41 & $(29.5)$ & \\
\hline Grade $1 \mathrm{~b}$ & - & & 30 & $(21.6)$ & \\
\hline Grade 2 & - & & 44 & $(31.7)$ & \\
\hline Grade 3 & - & & 3 & $(2.2)$ & \\
\hline
\end{tabular}

$p T$ pathological tumor status, $p N$ pathological nodal status, $p P$ pathological peritoneal metastasis, $C Y$ peritoneal cytology, pStage pathological stage, $R$ resection

${ }^{\mathrm{a}}$ Grade $0,100 \%$ residual tumor; Grade 1a, $>2 / 3$; Grade 1b, 1/3-2/3; Grade 2, <1/3; Grade 3, $0 \%$

${ }^{\mathrm{b}}$ Including three patients with pathological complete response

incidence of pCR may contribute to the survival benefit in the FLOT4 trial. The second possible reason is the difference in efficacy for SRC. In the FLOT4 trial, the survival was similar between SRC and non-SRC [18]. However, survival in SRC was considerably worse than that in non-SRC in the present study. SRC is considered to reflect resistance to chemotherapy [20,21]. Development of a regimen active 
Table 3 Site of first tumour recurrence

\begin{tabular}{lll}
\hline Recurrence site & Arm A $(n=89)$ & $\begin{array}{l}\text { Arm B } \\
(n=91)\end{array}$ \\
\hline Lymph node & 15 & 11 \\
Peritoneum & 68 & 73 \\
Distant & 15 & 11 \\
Other & 4 & 4 \\
\hline
\end{tabular}

for SRC is essential, since the proportion of SRC accounts for more than $60 \%$ of the cases with type 4 or large type 3 GC. The third possible reason is the low completion rate of planned treatment in the present study, which was $49.7 \%$ in Arm A and $47.0 \%$ in Arm B. There remains a possibility that intensive preoperative treatment adversely affected the response to adjuvant chemotherapy. However, the completion rate of adjuvant chemotherapy did not differ between the arms and was identical to that of the ACTS-GC trial [12]. Moreover, the completion rate in the NAC arm was superior to that reported in the MAGIC trial [16] and was almost identical to that observed in the FLOT-4 trial [18]. Thus, the possibility of deterioration of adjuvant chemotherapy efficacy by preoperative treatment appears to be unlikely.

One major difference between the FLOT and SP regimens is the inclusion of docetaxel, which has been reported to be active in not only perioperative chemotherapy but also adjuvant chemotherapy in GC. A recent randomized controlled trial (JACCRO GC-07) illustrated that postoperative chemotherapy comprising S-1 plus docetaxel (DS) improved RFS in patients with pathological stage III gastric cancer vs. S-1 monotherapy [22]. In addition, it has been reported that taxane-based therapy could be more effective against SRC in clinical and pre-clinical model [23, 24]. Thus, the efficacy of NAC regimens containing docetaxel requires further evaluation. We JCOG is now conducting a phase II trial of NAC using docetaxel, oxaliplatin and S-1 for extensive nodal disease [25]. Application of this regimen to type 4 or large type 3 can be possible as a future trial.

One limitation of this study was that all participants were Japanese. Greater toxicity has been reported for S-1 in Western patients than in Asians, and thus, a lower dosage is recommended for Western populations [26]. Therefore, it is unclear whether upfront surgery and postoperative S-1 are equally effective in Western patients.

\section{Conclusions}

Altogether, we concluded that NAC with SP is not recommended for type 4 or large type $3 \mathrm{GC}$. The OS and PFS of both arms in this study were considerably better than those reported previously. Thus, the standard treatment remains to be D2 surgery followed by adjuvant chemotherapy.

Acknowledgements This study was conducted by the Stomach Cancer Study Group of the Japan Clinical Oncology Group. The authors are grateful to the members of the JCOG Data Center and JCOG Operations Office for their support in this study. The study was supported in part by the National Cancer Center Research and Development Funds (23-A-19, 26-A-4, 29-A-3, 2020-J-3) and a Grant-in-Aid for Clinical Cancer Research (H19-15, H22-23, H22-27) from the Ministry of Health, Labor, and Welfare of Japan.

\section{Compliance with ethical standards}

Conflict of interest MT reports lecture fees from Taiho Pharmaceutical, Chugai Pharmaceutical, Ono Pharmaceutical, Bristol-Myers Squibb, Yakult Honsha, Takeda Pharmaceutical, Eli Lilly Japan KK, Pfizer Pharmaceutical, and Daiichi Sankyo outside the submitted work. JM reports personal fees from Chugai Pharmaceutical, outside the submitted work. HK reports personal fees from Johnson \& Johnson outside the submitted work. KN reports personal fees from Chugai, Bayer, and Taiho outside the submitted work. SI reports personal fees from Taiho Pharmaceutical, AstraZeneca, grants from Ono Pharmaceutical, and Merck Sharp \& Dohme outside the submitted work. TY reports personal fees from MSD, BMS, Ono, Taiho, Lilly, Chugai, Pfizer, Nihon Kayaku, TERUMO, Covidien, and Johnson \& Johnson outside the submitted work. NB received a research grant from Ono and Takeda and honorarium from Taiho, Ono, and Bristol-Myers Squibb outside the submitted work. TS reports personal fees from Taiho Pharma during the conduct of the study; personal fees from Taiho Pharma, Chugai Pharma, Ono Pharma, Eli Lilly, Yakult Pharma, and Daiichi Sankyo Pharma outside the submitted work. MS reports personal fees from Taiho Pharmaceutical during the study period. All other authors declare no competing interests.

Human rights statement and informed consent All procedures were in accordance with the ethical standards of the responsible committee on human experimentation (institutional and national) and with the 1964 Declaration of Helsinki and later versions. Informed consent for inclusion in the study was obtained from all patients.

\section{References}

1. Bray F, Ferlay J, Soerjomataram I, Siegel RL, Torre LA, Jemal A. Global cancer statistics 2018: GLOBOCAN estimates of incidence and mortality worldwide for 36 cancers in 185 countries. CA Cancer J Clin. 2018;68:394-424.

2. Cancer Registry and Statistics. Cancer Information Service, National Cancer Center, Japan. https://ganjoho.jp/reg_stat/stati stics/dl/index.html\#incidence4pref

3. Sasako M, Maruyama K, Kinoshita T. Neoadjuvant chemotherapy for gastric cancer: Indication and trial setting (in Japanese). Shokakigeka. 1992;15:159-67.

4. Pedrazzani C, Marrelli D, Pacelli F, Di Cosmo M, Mura G, Bettarini F, et al. Gastric linitis plastica: which role for surgical resection? Gastric Cancer. 2012;15:56-60.

5. Koizumi W, Tanabe S, Saigenji K, Ohtsu A, Boku N, Nagashima $\mathrm{F}$, et al. Phase I/II study of S-1 combined with cisplatin in patients with advanced gastric cancer. Br J Cancer. 2003;89:2207-12.

6. Iwasaki Y, Sasako M, Yamamoto S, Nakamura K, Sano T, Katai $\mathrm{H}$, et al. Phase II study of preoperative chemotherapy with S-1 and cisplatin followed by gastrectomy for clinically resectable 
type 4 and large type 3 gastric cancers (JCOG0210). J Surg Oncol. 2013;107:741-5.

7. Terashima M, Iwasaki Y, Mizusawa J, Katayama H, Nakamura K, Katai H, et al. Randomized phase III trial of gastrectomy with or without neoadjuvant S-1 plus cisplatin for type 4 or large type 3 gastric cancer, the short-term safety and surgical results: Japan Clinical Oncology Group Study (JCOG0501). Gastric Cancer. 2019;22:1044-52.

8. Japanese Research Society for Gastric Cancer. Japanese classification of gastric carcinoma-2nd English edition. Gastric Cancer. 1998;1:10-24.

9. Japanese Research Society for Gastric Cancer. Japanese classification of gastric carcinoma. 1st ed. Tokyo: Kanehara \& Co., Ltd.; 1995.

10. Lan KKG, DeMets DL. Discrete sequential boundaries for clinical trials. Biometrika. 1983;70:659-63.

11. Japanese Research Society for Gastric Cancer. Japanese Classification Of Gastric Carcinoma-2nd English edition-response assessment of chemotherapy and radiotherapy gor gastric carcinoma: clinical criteria. Gastric Cancer. 2001;4:1-8.

12. Sasako M, Sakuramot S, Katai H, Kinoshita T, Furukawa $\mathrm{H}$, Yamaguchi $\mathrm{T}$, et al. Five-Year outcomes of a randomized phase III trial comparing adjuvant chemotherapy with S-1 versus surgery alone in stage II or III gastric cancer. J Clin Oncol. 2011;29:4387-93.

13. Tomasello G, Petrelli F, Ghidini M, Pezzica E, Passalacqua R, Steccanella F, et al. Tumor regression grade and survival after neoadjuvant treatment in gastro-esophageal cancer: a meta-analysis of 17 published studies. Eur J Surg Oncol. 2017;43:1607-16.

14. Yamaguchi T, Takashima A, Nagashima K, Terashima M, Aizawa $\mathrm{M}$, Ohashi M, et al. Impact of pre-operative chemotherapy as initial treatment for advanced gastric cancer with peritoneal metastasis limited to peritoneal lavage cytology positive (CY1) or localized peritoneum metastasis (P1a): multi-institutional retrospective study. Submitted to Gastric Cancer. https://doi.org/10.1007/s1012 0-020-01137-6

15. Meguro Y, Yamaguchi H, Kitayama J, Kanamaru R, Matsumoto $\mathrm{S}$, Ui T, et al. Pathological complete response after intraperitoneal paclitaxel and systemic combined chemotherapy in a patient with peritoneal metastases from gastric cancer: a case report. Surg Case Rep. 2020;30(6):63-7.

16. Cunningham D, Allum W, Stenning S, Thompson JN, Van de Velde CJ, Nicolson M, et al. Perioperative chemotherapy versus surgery alone for resectable gastroesophageal cancer. N Engl J Med. 2006;355:11-20.

17. Al-Batran SE, Hofheinz RD, Pauligk C, Kopp HG, Haag GM, Luley $\mathrm{KB}$, et al. Histopathological regression after neoadjuvant docetaxel, oxaliplatin, fluorouracil, and leucovorin versus epirubicin, cisplatin, and fluorouracil or capecitabine in patients with resectable gastric or gastro-oesophageal junction adenocarcinoma (FLOT4-AIO): results from the phase 2 part of a multicentre, open-label, randomized phase 2/3 trial. Lancet Oncol. 2016;17:1697-708.

18. Al-Batran SE, Homann N, Pauligk C, Goetze TO, Meiler J, Kasper $\mathrm{S}$, et al. Perioperative chemotherapy with luorouracil plus leucovorin, oxaliplatin, and docetaxel versus fluorouracil or capecitabine plus cisplatin and epirubicin for locally advanced, resectable gastric or gastro-esophageal junction adenocarcinoma (FLOT4): a randomized, phase 2/3 trial. Lancet. 2019;393:1948-57.

19. Nakamura K, Kuwata T, Shimoda T, Mizusawa J, Katayama H, Kushima R, et al. Determination of the optimal cutoff percentage of residual tumors to define the pathological response rate for gastric cancer treated with preoperative therapy (JCOG1004-A). Gastric Cancer. 2015;18:597-604.

20. Piessen G, Messager M, Leteurtre E, Jean-Pierre T, Mariette C. Signet ring cell histology is an independent predictor of poor prognosis in gastric adenocarcinoma regardless of tumoral clinical presentation. Ann Surg. 2009;250:878-87.

21. Heger U, Blank S, Wiecha C, Langer R, Weichert W, Lordick F, et al. Is preoperative chemotherapy followed by surgery the appropriate treatment for signet ring cell containing adenocarcinomas of the esophagogastric junction and stomach? Ann Surg Oncol. 2014;21:1739-48.

22. Yoshida K, Kodera Y, Kochi M, Ichikawa W, Kakjeji Y, Sano T, et al. Addition of docetaxel to oral fluoropyrimidine improves efficacy in patients with stage III gastric cancer: interim analysis of JACCRO GC-07, a randomized controlled trial. J Clin Oncol. 2019;37:1296-304.

23. Pernot S, Voron T, Perkins G, Lagorce-Pages C, Berger A, Taieb J. Signet-ring cell carcinoma of the stomach: impact on prognosis and specific therapeutic challenge. World J Gastroenterol. 2015;21:11428-38.

24. Hultman B, Mahteme H, Sundbom M, Ljungman M, Larsson $\mathrm{R}$, Nygren P. Benchmarking of gastric cancer sensitivity to anticancer drugs ex vivo as a basis for drug selection in systemic and intraperitoneal therapy. J Exp Clin Cancer Res. 2014;33:110-20.

25. Sato Y, Kurokawa Y, Doki Y, Mizusawa J, Tanaka K, Katayama $\mathrm{H}$, et al. A Phase II study of preoperative chemotherapy with docetaxel, oxaliplatin and S-1 in gastric cancer with extensive lymph node metastasis (JCOG1704). Future Oncol. 2020;16:31-8.

26. Ajani JA, Rodriguez W, Bodoky G, Moiseyenko V, Lichinitser M, Gorbunova V, et al. Multicenter phase III comparison of cisplatin/S-1 with cisplatin/infusional fluorouracil in advanced gastric or gastroesophageal adenocarcinoma study: the FLAGS trial. J Clin Oncol. 2010;28:1547-53.

Publisher's Note Springer Nature remains neutral with regard to jurisdictional claims in published maps and institutional affiliations.

\section{Affiliations}

\section{Yoshiaki Iwasaki ${ }^{1} \cdot$ Masanori Terashima $^{2}$ - Junki Mizusawa ${ }^{3} \cdot$ Hiroshi Katayama $^{3} \cdot K^{\prime}$ Kenichi Nakamura ${ }^{3} \cdot$ Hitoshi Katai ${ }^{4} \cdot$ Takaki Yoshikawa $^{5} \cdot$ Seiji Ito $^{6}$ - Masahide Kaji ${ }^{7}$. Yutaka Kimura ${ }^{8}$. Motohiro Hirao ${ }^{9} \cdot$ Makoto Yamada $^{10}$. Akira Kurita $^{11}$. Masakazu Takagi ${ }^{12}$. Sang-Woong Lee ${ }^{13}$ - Akinori Takagane ${ }^{14}$ - Hiroshi Yabusaki ${ }^{15}$. Jun Hihara ${ }^{16}$. Narikazu Boku ${ }^{17} \cdot$ Takeshi Sano $^{18} \cdot$ Mitsuru Sasako $^{19}$}

1 Department of Surgery, IMS Tokyo Katsushika General Hospital, Tokyo, Japan

2 Division of Gastric Surgery, Shizuoka Cancer Center, 1007 Shimonagakubo, Nagaizumi, Shuzioka 411-8777, Japan
3 JCOG Data Center/Operations Office, National Cancer Center Hospital, Tokyo, Japan

4 Department of Gastric Surgery, National Cancer Center Hospital, Tokyo, Japan 
5 Department of Gastrointestinal Surgery, Kanagawa Cancer Center Hospital, Yokohama, Japan

6 Department of Gastroenterological Surgery, Aichi Cancer Center Hospital, Nagoya, Japan

7 Department of Surgery, Toyama Prefectural Central Hospital, Toyama, Japan

8 Department of Surgery, Sakai City Medical Center, Sakai, Japan

9 Department of Surgery, National Hospital Organization Osaka National Hospital, Osaka, Japan

10 Department of Surgery, Gifu Municipal Hospital, Gifu, Japan

11 Department of Surgery, National Hospital Organization Shikoku Cancer Center, Matsuyama, Japan

12 Department of Surgery, Shizuoka General Hospital, Shizuoka, Japan
13 Department of General and Gastroenterological Surgery, Osaka Medical College Hospital, Takatsuki, Japan

14 Department of Surgery, Hakodate Goryoukaku Hospital, Hakodate, Japan

15 Department of Surgery, Niigata Cancer Center Hospital, Niigata, Japan

16 Department of Surgery, Hiroshima City Asa Citizens Hospital, Hiroshima, Japan

17 Department of Gastrointestinal Medical Oncology, National Cancer Center Hospital, Tokyo, Japan

18 Department of Gastroenterological Surgery, Cancer Institute Hospital of Japanese Foundation for Cancer Research, Tokyo, Japan

19 Department of Surgery, Yodogawa Christian Hospital, Osaka, Japan 